\title{
Simulating the effect of alcohol on the structure of a membrane
}

\author{
Marieke Kranenburg, Berend Smit* \\ Department of Chemical Engineering, University of Amsterdam, Nieuwe Achtergracht 166, 1018 WV Amsterdam, The Netherlands
}

Received 6 April 2004; accepted 13 April 2004

Available online 18 May 2004

Edited by Sandro Sonnino

\begin{abstract}
Adsorption of alcohol molecules or other small amphiphilic molecules in the cell membrane can induce significant changes in the structure of the membrane. To understand the molecular mechanisms underlying these structural changes, we developed a mesoscopic membrane model. Molecular simulations on this model nicely reproduce the experimental phase diagrams. We find that alcohol can induce an interdigitated structure in which the normal bilayer structure changes into a monolayer in which the alcohol molecules screen the hydrophobic tails from the water phase. We compute the effect of the chain length of the alcohol on the phase behaviour of the membrane. At low concentrations of alcohol, the membrane has domains of the interdigitated phase that are in coexistence with the normal membrane phase. We use our model to clarify some of the experimental questions related to the structure of the interdigitated phase and put forward a simple model that explains the alcohol chain length dependence of the stability of this interdigitated phase.
\end{abstract}

(C) 2004 Published by Elsevier B.V. on behalf of the Federation of European Biochemical Societies.

Keywords: Membrane structure; Interdigitation; Molecular simulations; Mesoscopic model; Phase behaviour

\section{Introduction}

The generally accepted mechanism for the activity of drugs is that they specifically bind to specific sites at a target protein and influence the functioning of a protein. With this model, it is difficult to explain the effects of anaesthesia or alcohol on the functioning of an organism [1]. Because anaesthesia and alcohols are relatively small molecules, it is unlikely that there are specific sites in proteins to which these molecules bind [1]. Therefore, mechanisms have been put forward that these small molecules adsorb in a membrane and influence its structure $[2,3]$. These structural changes of the membrane can influence the conformation of proteins or other structures embedded in the membrane. This provides an indirect route in which alcohol or other small amphiphilic molecules influence the function of a cell. Experiments on phospholipid membranes have shown that alcohol can, for example, induce the interdigitated phase [4] (Fig. 1). This is an extreme example of the effect alcohol can have on the structure of a membrane. In an ordinary membrane the hydrophobic thickness is approximately twice the

\footnotetext{
* Corresponding author. Fax: +31-020-5255604.

E-mail address: B.Smit@science.uva.nl (B. Smit).
}

Abbreviations: DPD, dissipative particle dynamics; DSPC, distearoylphosphatidylcholine length of the hydrophobic tails of a phospholipid, in the interdigitated phase the hydrophobic thickness is reduced to the sum of the length of the hydrophobic tails of a single phospholipid and the alcohol. That small molecules can have such a dramatic effect on the structure of a membrane rationalizes the hypothesis of Cantor [2] that small molecules can induce structural changes of the environment of a membrane protein or ion channel. Understanding these induced structural changes is therefore not only of fundamental interest but might also give us a better understanding of the functioning of cell membranes.

Fig. 1 shows the commonly assumed structure of this interdigitated phase, in which the alcohol molecules prevent the hydrophobic tails of the lipids to be exposed to water $[5,6]$. This model nicely explains the experimentally observed hydrophobic thickness and provides a simple molecular explanation as to why the alcohol molecules stabilize the interdigitated phase and why it is often assumed that in the interdigitated phase the optimal alcohol to lipid ratio in the membrane is $2: 1$. At this point, it is important to mention that one cannot measure the concentration of alcohol in the membrane directly and therefore there is no direct experimental evidence for this $2: 1$ ratio. Therefore, very little is known about the limiting concentration of alcohol in the membrane to induce interdigitation and how this concentration depends on the chain length of the alcohol [7].

These experimental uncertainties motivated us to use molecular simulation to study the effect of alcohol. State of the art molecular dynamics simulations give detailed structural information on the position of these molecules [8,9], but are too time consuming to observe structural changes in the membrane. As an alternative, we use a hierarchical approach in which realistic all-atom simulations are used to determine the effective interaction parameters of a mesoscopic model. At this mesoscopic level, simulations are 4-5 orders of magnitude more efficient $[10,11]$, allowing us to compute complete phase diagrams. Whereas previous mesoscopic simulations were aimed at qualitative insights, we demonstrate here that such a hierarchical mesoscopic model yields quantitative predictions of the structure of a membrane.

\section{Model and computational details}

We distinguish three types of particles, $w, h$, and $t$, to mimic water and the head- and tail-atoms of a lipid, respectively. The hydrophilic and hydrophobic particles interact via a soft-repulsion model commonly used in dissipative particle dynamics (DPD) [12,13]. In a DPD simulation, a particle represents the centre of mass of a cluster of 


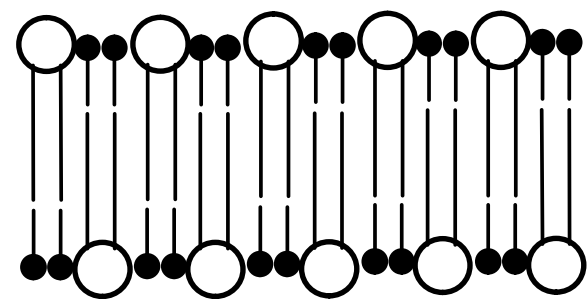

Fig. 1. Schematic structure of the interdigitated phase in which two alcohol molecules pair up with one phospholipid.

atoms. The total force on such a particle consists of dissipative, random, and conservative forces. The dissipative and random forces are chosen such that a proper canonical distribution is sampled [13]. For the conservative force, we use the conventional soft-repulsive interaction, given by

$F_{i j}^{\mathrm{C}}=\left\{\begin{array}{cc}a_{i j}\left(1-r_{i j} / r_{\mathrm{c}}\right) & r_{i j}<r_{\mathrm{c}}, \\ 0 & r_{i j} \geqslant r_{\mathrm{c}},\end{array}\right.$

where $r_{i j}$ is the distance between particles $i$ and $j, a_{i j}$ is the parameter characterizing the interaction between two particles, and $r_{\mathrm{c}}$ is the cutoff radius. The DPD parameters are related to the compressibility of water and to Flory-Huggins solubility parameters such that a reasonable description of the thermodynamics of the real system can be obtained [12]

We consider the phospholipid distearoyl-phosphatidylcholine (DSPC). We assume that a DPD particle occupies a volume of $90 \AA^{3}$, which results in a mapping in which DSPC is represented by three hydrophilic head beads and two seven beads tails $\left(\mathrm{h}_{3}\left(\mathrm{t}_{7}\right)_{2}\right)$. For a correct description of the experimental chain length dependence of the area per lipid, it is essential to properly reproduce the conformations of the lipid. Molecular Dynamics simulations of a single phospholipid in water using a realistic all-atom representation are used to generate configurations of the lipid, which were subsequently used to optimize the intramolecular interactions (bond-bending and bond-vibration) of the DPD model. We used similar parameters as Groot [12] for the softrepulsions in the conservative DPD interactions $\left(a_{\mathrm{ww}}=a_{\mathrm{tt}}=25\right.$, $a_{\mathrm{ht}}=a_{\mathrm{wt}}=80, a_{\mathrm{hh}}=35$, and $a_{\mathrm{hw}}=15$ ), the intramolecular interactions include a bond-bending potential $U_{\theta}=(1 / 2) k_{\theta}\left(\theta-\theta_{0}\right)^{2}$, with $k_{\theta}=6$ and $\theta_{0}=180^{\circ}$ between three subsequent beads in the tails and $k_{\theta}=6$ and $\theta_{0}=90^{\circ}$ for the vectors connecting the first bead of the tails with the headgroup. Two consecutive beads are connected by harmonic springs with spring constant $k_{r}=100$ and equilibrium distance $r_{0}=0.7$.

The coarse-graining procedure of Groot can be used at any temperature, that is, if we are interested at higher temperature we can again match the compressibility and Flory-Huggins solubility parameters at the temperature of interest. This leads to temperature dependent parameters which make the interpretation of the results more difficult. To avoid these difficulties, we have used the experimental phase transition temperatures (without alcohol) to relate the DPD energy and temperature scales to the experimental system. The length scale follows from the assumption that a DPD particle occupies a volume of $90 \AA^{3}$. With these parameters our simulations predict an area per surfactant of $69 \mathrm{~A}^{2}$ of the membrane in the $\mathrm{L}_{\alpha}$ or fluid phase, independent of the chain length. This is in good agreement with the experimental values which are in the range $58-72 \AA^{2}$.

A biological membrane is not subject to external constraints and therefore adopts a configuration which is tensionless. Lipowski and coworkers [14] emphasize the importance of simulating at exactly the area for which the interfacial tension is zero and determine this area iteratively. We use a different approach in which we mimic the experiment by simulating an ensemble in which we impose the interfacial tension. After a randomly selected number of DPD steps, we perform a Monte Carlo move in which we change the area of our bilayer in such a way that the total volume of the system remains constant. This move is accepted with a probability [15]

$\operatorname{acc}(\mathrm{o} \rightarrow \mathrm{n})=\min \left(1, \frac{\exp \left\{-\beta\left[U(\mathrm{n})-\gamma A_{\mathrm{n}}\right]\right\}}{\exp \left\{-\beta\left[U(\mathrm{o})-\gamma A_{\mathrm{o}}\right]\right\}}\right)$,

where $U(\mathrm{o})$ and $U(\mathrm{n})$ indicate the energy of the old and the new configuration, respectively, $\gamma$ the interfacial tension, $A$ the area of the bilayer and $\beta=1 / k_{\mathrm{B}} T$. To obtain the tensionless state of the bilayer, $\gamma$ is set to zero to ensure that the membrane adopts the equilibrium area per lipid $[16,17]$. The importance of this method is that it allows us to observe directly phase transitions in which the area per lipid changes.

We have used a system of 200 phospholipids and 20-200 alcohols initially randomly distributed in the simulation box. 3200-6700 water
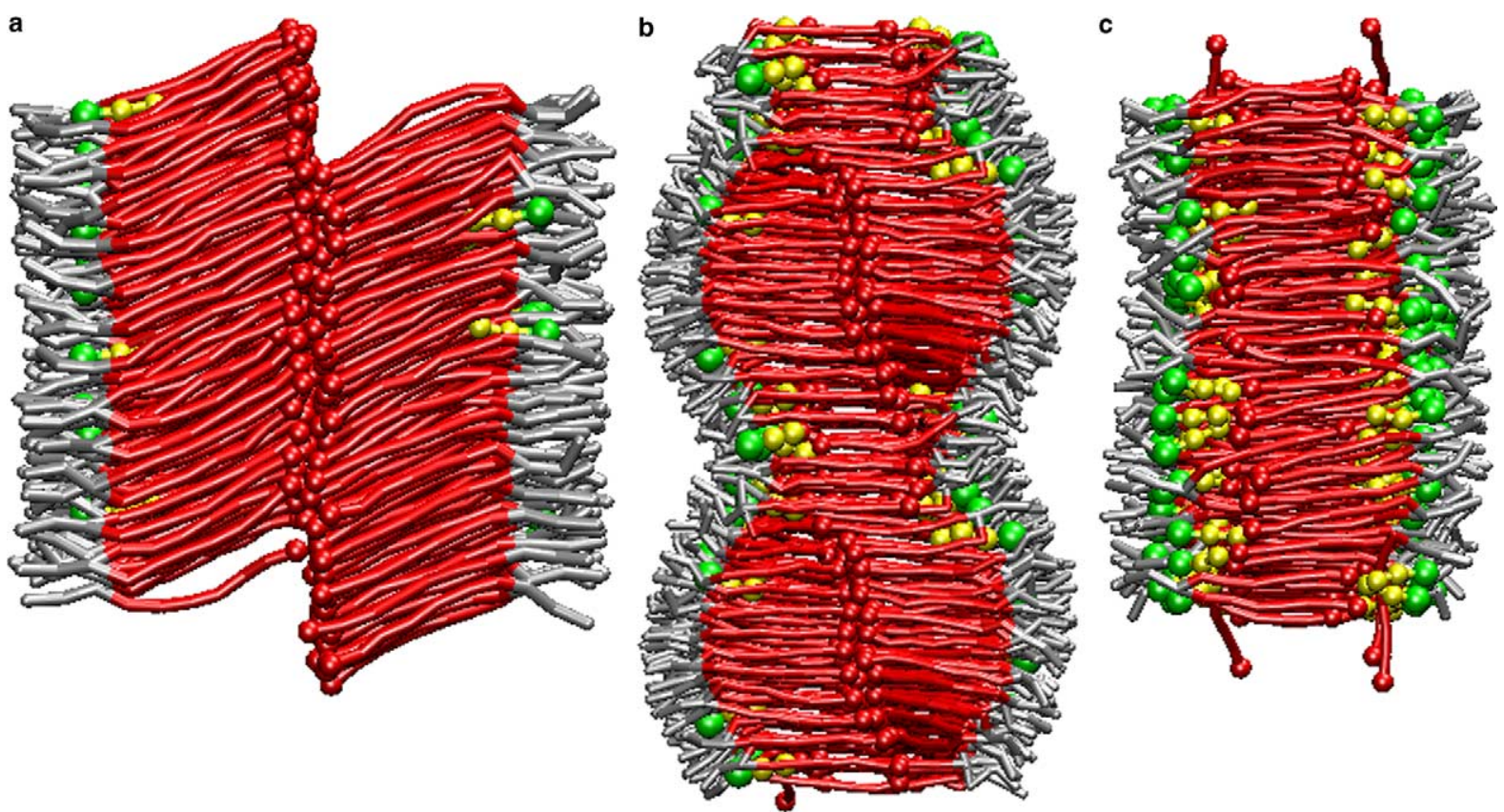

Fig. 2. Snapshots of a bilayer in (a) $\mathrm{L}_{\mathrm{c}}$ phase, (b) coexistence between the interdigitated and $\mathrm{L}_{\beta^{\prime}}$ phase, and (c) interdigitated phase ( $\left.\mathrm{L}_{\beta \mathrm{I}}\right)$ consisting of the lipid $h_{3}\left(t_{7}\right)_{2}$ with various concentrations of the model alcohol ht $t_{2}$. The bonds represent the lipids, where grey is the hydrophilic head group and red the tails. The bonds and spheres indicate the alcohol molecules, where green is the hydrophilic head group and yellow the tail. The terminal beads of both the lipid and the alcohol are depicted by a larger sphere. In (b) the system is depicted twice, so that the coexistence is illustrated more clearly. 

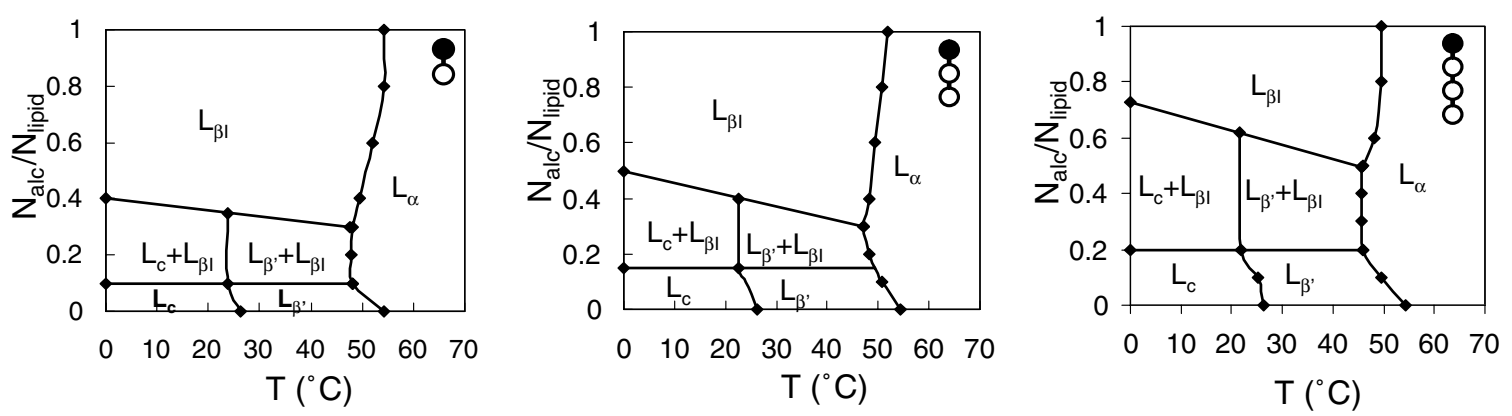

Fig. 3. Phase diagrams of the lipid $h_{3}\left(t_{7}\right)_{2}$ with the alcohols ht (left), $h_{2}$ (middle), and ht ${ }_{3}$ (right).

particles were added to ensure a fully hydrated bilayer, which was formed by self assembly. The overall density of the system was $\rho=3$. A typical simulation required 100000 cycles, of which 20000 cycles are equilibration.

\section{Results and discussion}

The effect of alcohol on the structure and phase behaviour is shown in Figs. 2 and 3, respectively. At zero alcohol concentration, we nicely recover the experimentally observed phases. At low temperatures we observe the $\mathrm{L}_{\mathrm{c}}$ phase, in which the tails are ordered and tilted. Increasing the temperature causes the tails to lose some of their order in the $\mathrm{L}_{\beta^{\prime}}$ phase and eventually also their tilt in the disordered fluid or $\mathrm{L}_{\alpha}$ phase. At low concentrations of alcohol, the molecules are homogeneously distributed in the gel phases, $\mathrm{L}_{\beta^{\prime}}$ or $\mathrm{L}_{\mathrm{c}}$ phase. At high alcohol concentrations, we find the interdigitated phase $\mathrm{L}_{\beta \mathrm{I}}$. Interestingly, in between we find a region of coexistence of the interdigitated and non-interdigitated phase, in which the alcohols are mainly located in the interdigitated part. The simulations reproduce the so-called "biphasic effect" [18]; at low alcohol concentrations the main transition, from the gel phase to the fluid phase, shifts to a lower temperature, while at high concentrations it shifts to a higher temperature. This behaviour is directly related to the effect of the alcohol on the stability of the low temperature phases [19]; the $\mathrm{L}_{\beta^{\prime}}$, phase is destabilized by alcohol, while the $\mathrm{L}_{\beta \mathrm{I}}$ can easily incorporate alcohol molecules.

That such a simple lipid/water/alcohol model nicely reproduces the experimental phase diagram and the biphasic effect gives us confidence that we can also use our model to compare those properties for which the experimental data are less conclusive. Adachi et al. [6] proposed a model of the structure of the bilayer in which the terminal methyl group of the alcohol faces a terminal methyl group of a lipid chain (see Fig. 1). This model is based on the experimental observation that the membrane thickness increases by about $0.08 \mathrm{~nm}$ per one methylene unit in either the alcohol molecules or the phospholipids [5,6] and that two alcohol molecules can occupy a volume surrounded by the PC head groups of one layer. Hence, the number of alcohol molecules in the membrane is twice the number of lipids, independently of the length of the alcohol. This conclusion clearly differs with our results in which the stability of the interdigitated phase depends on the length of the alcohol (see Fig. 3). Fig. 4 shows that the computed hydrophobic thickness $D_{\mathrm{c}}$ for any combinations of lipids and alcohols is in surprisingly good quantitative agreement with the experimental data $[5,6,20]$. For all combinations we compute a linear relation between $D_{\mathrm{c}}$ and the total number of beads, not only for an alcohol:lipid ratio of 2:1 but also for much lower alcohol concentrations in the membrane. The fully interdigitated phase occurs at much lower number of alcohols than twice the number of lipids in the lipid bilayer and it decreases with chain length.

The incorporation of alcohols at the membrane interface creates energetically unfavourable voids in the hydrophobic core of the $\mathrm{L}_{\beta^{\prime}}$ phase (see Fig. 5). In the interdigitated phase, the tail ends of the lipid are in contact with the interface. Adding alcohol reduces these energetically unfavourable interactions. Clearly at the 2:1 ratio, the energy of the interdigitated phase will be lowest, but at much lower alcohol concentrations the interdigitated phase can already be more stable than the $\mathrm{L}_{\beta^{\prime}}$ phase. We can now also understand the dependence of the stability of the $\mathrm{L}_{\beta \mathrm{I}}$ phase on alcohol length. The longer the alcohol, the smaller the voids in the alcohol saturated $\mathrm{L}_{\beta^{\prime}}$ phase, hence these longer alcohol molecules perturb the $\mathrm{L}_{\beta^{\prime}}$ phase to a lesser extent. For the interdigitated phase, longer alcohol results in a thicker hydrophobic core. A

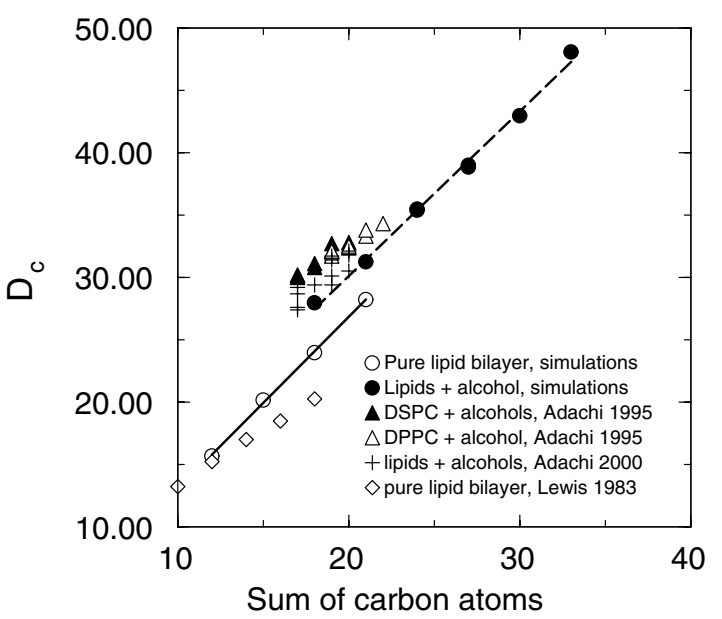

Fig. 4. Comparison of the experimentally measured thickness of the hydrophobic core $D_{\mathrm{c}}$ as a function of the total number of beads of the lipid and the alcohol. The length of alcohol tails ranges from 1 to 3 and the length of the lipid tails from 6 to 8 beads. The hydrophobic thickness of the pure lipid bilayer is divided by two. All thicknesses are measured at a fully interdigitated bilayer, in which the ratio $N_{\text {alc }} / N_{\text {lipid }}=1: 1$. The dashed lines are the best fits of the pure lipid system and the mixtures of lipids and alcohols. We only show the results for this ratio, similar results have been obtained for lower alcohol concentrations. 


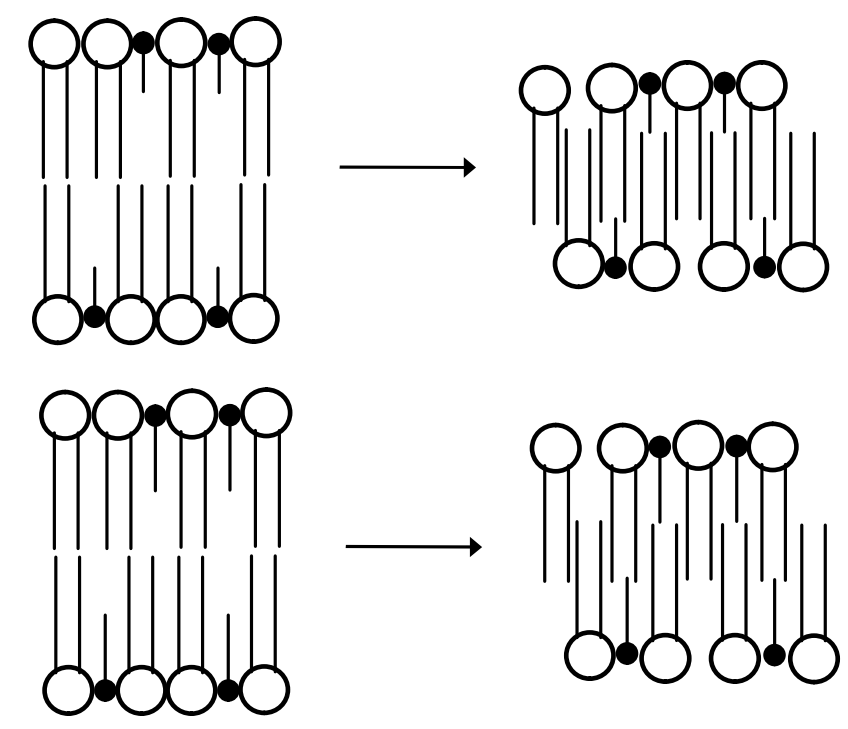

Fig. 5. Schematic drawing of the formation of the interdigitated phase. (right) Voids between the hydrophobic tails destabilize the gel phases. (left) With increasing tail length of the alcohol the voids become smaller in the $\mathrm{L}_{\beta^{\prime}}$ phase, while in the interdigitated phase the part of the lipid tails exposed to water becomes larger. As the chain length of the alcohol increases the perturbation of the $\mathrm{L}_{\beta^{\prime}}$ phase becomes smaller, while for the interdigitated phase the voids are larger.

void in this phase exposes more hydrophobic groups of the lipids to the water phase. These combined effects explain why the longer the tail length of the alcohol, the more alcohol is needed to stabilize the $\mathrm{L}_{\beta \mathrm{I}}$ phase. Similarly, we can understand why a higher concentration of alcohol is needed before the $L_{\beta^{\prime}}$ phase is destabilized by a longer alcohol.

That a mesoscopic model can be developed systematically which not only gives us qualitative insights into the molecular mechanisms that are responsible for alcohol induced changes in the structure of a membrane, but also gives quantitative predictions on the dramatic effects of alcohol on the structure of a membrane is an important conclusion of this work. It would be interesting to investigate the role of "impurities" such as peptides, proteins, or membrane channels in these systems. This might shed some light on the controversy related to the mechanism of how such small molecules can influence the functioning of organisms.

Acknowledgements: The authors acknowledge financial support of the Dutch Science Foundation (NWO).

\section{References}

[1] Sonner, J.M. et al. (2003) Anesth. Analg. 97, 718-740.

[2] Cantor, R.S. (2003) Biochemistry 42, 11891-11897.

[3] Cantor, R.S. (1997) Biochemistry 36, 2339-2344.

[4] Slater, J.L. and Huang, C.H. (1988) Prog. Lipid Res. 27, 325-359.

[5] Adachi, T. (2000) Chem. Phys. Lipids 107, 93-97.

[6] Adachi, T., Takahashi, H., Ohki, K. and Hatta, I. (1995) Biophys. J. 68, 1850-1855.

[7] Löbbecke, L. and Cevc, G. (1995) Biochim. Biophys. Acta 1237, 59-69.

[8] Koubi, L., Saiz, L., Tarek, M., Scharf, D. and Klein, M.L. (2003) J. Phys. Chem. B 107, 14500-14508.

[9] Tang, P. and Xu, Y. (2002) Proc. Natl. Acad. Sci. USA 99, 1603516040.

[10] Groot, R.D. and Rabone, K.L. (2001) Biophys. J. 81, 725-736.

[11] Shelley, J.C., Shelley, M.Y., Reeder, R.C., Bandyopadhyay, S., Moore, P.B. and Klein, M.L. (2001) J. Phys. Chem. B 105, 97859792.

[12] Groot, R.D. and Warren, P.B. (1997) J. Chem. Phys. 107, 44234435.

[13] Frenkel, D. and Smit, B. (2002) Understanding Molecular Simulations: from Algorithms to Applications. Academic Press, San Diego.

[14] Goetz, R., Gompper, G. and Lipowsky, R. (1999) Phys. Rev. Lett. $82,221-224$

[15] Venturoli, M. and Smit, B. (1999) Phys. Chem. Commun. 10.

[16] Kranenburg, M., Venturoli, M. and Smit, B. (2003) J. Phys. Chem. B 107, 11491-11501.

[17] Kranenburg, M., Venturoli, M. and Smit, B. (2003) Phys. Rev. E 67, art. no.-060901.

[18] Rowe, E.S. (1983) Biochemistry 22, 3299-3305.

[19] Simon, S.A. and McIntosh, T.J. (1984) Biochim. Biophys. Acta $773,169-172$

[20] Lewis, B.A. and Engelman, D.M. (1983) J. Mol. Biol. 166, 211217. 